\title{
Why Peer Review?
}

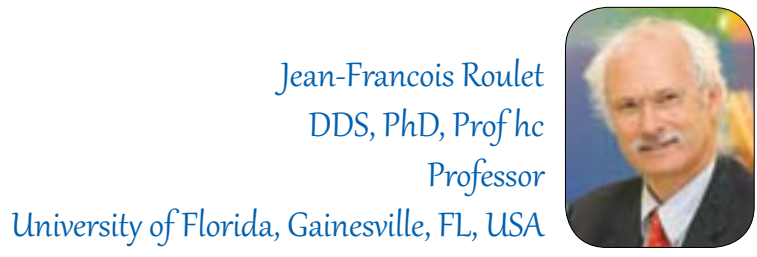

Dear Readers,

As dentists we are part of the medical community. We are the experts in everything related to the health of the oral cavity and its surroundings. Therefore ethical guidelines require us to deliver treatment or provide advice of the highest quality for the benefit of our patients. This sounds great; however it includes an inherent conflict: how to define quality. This is very difficult in medicine and dentistry. Quality may be divided into process quality (in simple terms: do the right thing) and outcome quality (in simple terms: do it right). Both need definitions, what is good or bad; and this is where the problem sits.

Once upon a time our teachers were setting the requirement for what is considered good quality, often based on their opinion, and we students had to comply. These days things got more complicated. We need to base our definitions on facts or results based on experiments. For dental care and medicine the ultimate measurement of good quality is the survival of the restoration or the patient after an intervention or therapy. "Evidence based" is the magic word here. However we cannot base all our doing only on results of clinical studies, as we would postpone good treatment options to our patients for years. Therefore we need to accept lower evidence levels such as in vitro studies as well, to make up our mind.

In the age of the internet information is available instantaneously and globally, which is a very good thing. The back side of this is the information overload and the black side is that the average user cannot distinguish anymore which information is relevant or true, or which information is pure claim or just intended to motivate the target reader to use it, or to use the product described. This is where peer review becomes important. Anders Linde, the Editor of the European Journal of Oral Sciences once stated: "Nothing is scientifically shown or proven before it has been published in a scientific journal with a peer review system, so one can critically judge what was done, how it was done and evaluate how solid it is." The application of this by an editorial team means that a group of experts in the field (the peers) will have very carefully looked at every document which is finally published. They will check if the information provided is new, if the formatting is correct, if the language is used correctly, if the methods used make sense and are free of bias. Statisticians will look at the results to make sure that the outcome is really a function of the experimental variables. The experts will also ask themselves "Does it make sense?" and will critically look at results which may significantly differ from other similar tests. Finally, the editorial team will make sure that the conclusions drawn are strictly related to the outcome of the experiments. If there are questions, which is almost always the case, then the authors are challenged to address them. These are a few facts that make the difference between a non peer reviewed publication and a peer reviewed publication. Of course during the review process some manuscripts get rejected. These are the ones that do not fulfill the quality requirements or do not survive the critical review because of incurable flaws (mostly in the methodology). Notwithstanding, the main objective of the review process is to improve the quality of the manuscript, so you, readers, can trust the information provided.

So, in order to be credible, there is no alternative to peer review! 